Special Section

\title{
Bodies in Pain, Pleasure, and Flux Transgressive Femininity in Japanese Media and Literature
}

Edited by

Marianne Tarcov and Fareed Ben-Youssef 
